\title{
Resposta da goiabeira à lâmina de água e à adubação nitrogenada ${ }^{1}$
}

\author{
José L. Maciel ${ }^{2}$, José Dantas Neto ${ }^{3} \&$ Pedro D. Fernandes ${ }^{3}$
}

\begin{abstract}
RESUMO
Estudar os efeitos da irrigação e da adubação nitrogenada via irrigação na produtividade da goiabeira cultivar Paluma, com quatro anos de idade, sob sistema de microaspersão no espaçamento $6 \times 5 \mathrm{~m}$, foi o principal objetivo dos autores com este trabalho. O experimento foi conduzido na Estação Experimental de Veludo, município de Itaporanga, PB, no delineamento experimental em blocos casualizados, com quatro repetições, em esquema fatorial $4 \times 4$, cujos fatores foram: quatro lâminas de irrigação, $L_{1}=0,60 L_{b}, L_{2}=1,00 L_{b}, L_{3}=1,40 L_{b}$ e $L_{4}=1,80 L_{b}$, sendo $L_{b}$ a lâmina bruta de irrigação, e quatro níveis de nitrogênio, $N_{1}=50, N_{2}=100, N_{3}=150$ e $N_{4}=200 \mathrm{~kg} \mathrm{ha}^{-1}$. As irrigações foram realizadas diariamente durante o ciclo da cultura (198 dias) após poda de frutificação e as fertirrigações o foram na freqüência de três aplicações semanais, totalizando 80 aplicações; as quantidades totais de água (irrigação + precipitação) por tratamento, foram $\mathrm{L}_{1}=1.144 ; \mathrm{L}_{2}=1.465 ; \mathrm{L}_{3}=1.785$ e $\mathrm{L}_{4}=2.106 \mathrm{~mm}$, respectivamente. A produtividade máxima econômica foi de $35.594 \mathrm{~kg} \mathrm{ha}^{-1}$ correspondente à lâmina de água $1.721 \mathrm{~mm}$ e de $167 \mathrm{~kg} \mathrm{ha}^{-1}$ de nitrogênio resultando em uma receita líquida de $R \$ 13.154,68 \mathrm{ha}^{-1}$, considerando-se o custo da água de $\mathrm{R} \$ 0,65 \mathrm{~mm} \mathrm{ha}^{-1}$, nitrogênio de $\mathrm{R} \$ 1,15 \mathrm{~kg}^{-1}$ e o preço de venda do produto $(\mathrm{Py})$ de $\mathrm{R} \$ 0,50 \mathrm{~kg}$.
\end{abstract}

Palavras-chave: fruticultura, manejo de irrigação, análise econômica

\section{Response function of the guava to water depth and nitrogen fertilization}

\begin{abstract}
The objective of this research was to study the effects of irrigation and nitrogen fertilization applied by fertigation on the productivity of the guava, Paluma variety, four years old, under microirigation system with $6 \times 5 \mathrm{~m}$ spacing. The experiment was carried out at the Experimental Station of Veludo, in Itaporanga city, PB. The experimental design was randomized blocks, in a $4 \times 4$ factorial scheme with four repetitions, whose factors were: four irrigation levels, $L_{1}=0.60 L_{b}$, $L_{2}=1.00 L_{b}, L_{3}=1.40 L_{b}$ and $L_{4}=1.80 L_{b}$ and four levels of nitrogen, $N_{1}=50, N_{2}=100, N_{3}=150$ and $\mathrm{N}_{4}=200 \mathrm{~kg} \mathrm{ha}^{-1}$. The irrigation was accomplished daily during the crop cycle (198 days) after frutification pruning. The fertigation was performed three days a week during the crop cycle (80 applications). The effects of the treatments were evaluated by production, number of fruits per plant, total weight of fruits per plant and mean weight of fruits. The maximum economic productivity for the two factors $(\mathrm{LxN})$ was $35.594 \mathrm{~kg} \mathrm{ha}^{-1}$ with the application of $\mathrm{L}=1.721 \mathrm{~mm}$ and $\mathrm{N}=167 \mathrm{~kg} \mathrm{ha}^{-1}$, with total income of $R \$ 13,154.68 \mathrm{ha}^{-1}$, considering the water cost of $R \$ 0.65 \mathrm{~mm} \mathrm{ha}^{-1}$ and nitrogen cost of $R \$ 1.15 \mathrm{~kg}^{-1}$ and sale price $P y=R \$ 0.50 \mathrm{~kg}$.
\end{abstract}

Key words: fruit culture, irrigation management, economic analysis

1 Parte da tese de Doutorado do primeiro autor apresentada à UAEAg/UFCG

2 EBDA, PÇ. Imaculada Conceição 28, Centro, CEP 48900-000 Juazeiro, BA. Fone: (74) 3611-7666. E-mail: jotalinsm@yahoo.com.br

3 UAEAg/UFCG, CEP 58109-970, Campina Grande, PB. Fone: (83) 3310-1373. E-mail: dantasneto@pesquisador.cnpq.br; pdantas@deag.ufcg.edu.br 


\section{INTRODUÇÃO}

São muitas as vantagens econômicas e sociais da fruticultura irrigada, como elevação do nível de emprego, fixação do homem no campo, melhor distribuição da renda regional, geração de produtos de alto valor comercial e expressivas receitas e impostos, além de boas expectativas de mercado interno e externo, gerando divisas. Em 2004 foram produzidas em torno de $920.000 \mathrm{t}$ de frutas diversas com receita bruta superior a $\mathrm{R} \$ 200$ milhões de reais e geração de mais de 50.000 empregos diretos e indiretos (CODEVASF, 2005).

Dentre as culturas exploradas são mais de 40 espécies, destacando-se manga, uva, coco e banana. Entre as novas alternativas se encontra a cultura da goiaba, atividade de rentabilidade viável e com possibilidade de expansão no País. Originária de regiões de clima tropical e subtropical, a goiabeira (Psidium guajava L.) vegeta e produz, satisfatoriamente, do nível do mar até a altitude de $1.700 \mathrm{~m}$; é uma planta relativamente resistente à seca, exige temperatura média anual superior a $22{ }^{\circ} \mathrm{C}$ e se adapta melhor em solos arenoargilosos, profundos, bem drenados, com faixa de $\mathrm{pH}$ entre 5,0 e 6,5 (Gonzaga Neto, 2001). Dentre as cultivares podese citar a Pedro Sato, a Rica e a Paluma, de aptidões mistas, muito utilizadas para renovação ou implantação de novos pomares, com destino prioritário à indústria, especialmente as duas últimas, sendo a 'Paluma' a mais difundida no Brasil, alcançando produtividade superior a $50 \mathrm{t} \mathrm{ha}^{-1} \mathrm{ano}^{-1}$ (Gonzaga Neto, 2001; Lima et al., 2002).

A região semi-árida paraibana, sobretudo os vales úmidos dos rios Piancó, Piranhas e do Peixe, evidencia potencialidade para instalação de culturas nobres, como a videira, mangueira e goiabeira, dentre outras, devido às condições favoráveis de solo e clima, fatores esses requeridos para implantação de grandes projetos agrícolas com frutíferas, a exemplo do Vale do São Francisco. As fruteiras encontram, quando irrigadas, condições essenciais para desenvolver todo o seu potencial produtivo, proporcionando rendimentos adequados e maior retorno econômico para o fortalecimento da agricultura familiar (Gonzaga Neto, 2001).

No manejo dos fertilizantes em fruteiras tropicais devese levar em consideração a necessidade da cultura, a disponibilidade de nutrientes no solo, o modo e o custo de distribuição do fertilizante no campo, o parcelamento, de acordo com as fases de desenvolvimento da planta e a preservação do meio ambiente (Papadopoulos, 2001; Lopez, 2001).

O lucro, juntamente com o valor social, constitui o principal objetivo da empresa agrícola; portanto, o uso racional dos recursos disponíveis no processo de produção, de forma a se obter os mais altos níveis de rendimento econômico, deve ser considerado; os maiores valores no rendimento de uma cultura dependem dos fornecimentos adequados de água e de fertilizantes (Aguiar, 2005); portanto, o uso das funções de resposta das culturas constitui fonte valiosa de informações a ser utilizada nos modelos de tomada de decisão, visando à otimização do uso dos fatores envolvidos na produção.

Algumas pesquisas foram realizadas para se estudar os efeitos de lâminas de água (Sousa, 1997; Bassoi et al., 2002), e os efeitos de nutrientes (Martinez Júnior \& Pereira, 1986), sobre a produtividade de várias cultivares de goiabeira em solos e climas variados porém não se constatou qualquer registro de estudos envolvendo os efeitos conjunto de água e nutrientes sobre o rendimento desta cultura.

Objetivou-se, através deste trabalho, determinar os níveis máximos econômicos de água e nitrogênio que proporcionem a máxima receita líquida da goiabeira Paluma.

\section{MATERIAL E MÉTODOS}

O experimento foi conduzido em um pomar cultivado de goiabeira (Psidium guajava L.), cultivar Paluma, espaçamento de $6 \times 5 \mathrm{~m}$, com quatro anos de idade, localizado na Estação Experimental de Veludo, pertencente à Empresa Estadual de Pesquisa Agropecuária da Paraíba-EMEPA, município de Itaporanga, $\mathrm{PB}$, cujas coordenadas geográficas são $7^{\circ} 18^{\prime}$ de latitude Sul e 38 9' de longitude oeste, e altitude $291 \mathrm{~m}$, a aproximadamente $300 \mathrm{~km}$ de Campina Grande, PB.

O clima da região, de acordo com a classificação de Köeppen, é do tipo Aw', quente e seco com chuvas de verão e de outono, precipitação média anual de 806 mm, concentrada nos meses de janeiro a abril; temperatura média anual em geral superiore a $24{ }^{\circ} \mathrm{C}$ e a umidade relativa do ar da ordem de $74 \%$, em relação ao solo, apresenta textura franco-argilosa nas camadas de 0-0,20 e 0,20-0,40 m, cujas características físico-hídricas e químicas determinadas no Laboratório de Irrigação e Salinidade da Universidade Federal de Campina Grande, são observadas nas Tabelas 1 e 2, respectivamente.

Estudaram-se dois fatores de produção: a) lâmina bruta de irrigação $\left(\mathrm{L}_{\mathrm{b}}\right)$, determinada com base na evaporação diária do tanque classe A (Et) e no coeficiente da cultura (kc) (Eq. 1),

Tabela 1. Características físico-hídricas do solo cultivado com goiabeira cultivar Paluma, com quatro anos de idade. Itaporanga, PB, 2005

\begin{tabular}{|c|c|c|}
\hline \multirow{2}{*}{ Características } & \multicolumn{2}{|c|}{ Profundidade (m) } \\
\hline & $0-0,20$ & $0,20-0,40$ \\
\hline \multicolumn{3}{|c|}{ Físicas } \\
\hline \multicolumn{3}{|l|}{ Granulometria (\%) } \\
\hline Areia & 41,89 & 44,61 \\
\hline Silte & 26,92 & 28,15 \\
\hline Argila & 31,19 & 27,24 \\
\hline Classificação textural & Franco-argilosa & Franco-argilosa \\
\hline Densidade solo $\left(\mathrm{g} \mathrm{cm}^{-3}\right)$ & 1,29 & 1,27 \\
\hline Densidade das partículas $\left(\mathrm{g} \mathrm{cm}^{-3}\right)$ & 2,77 & 2,76 \\
\hline Porosidade total (\%) & 53,42 & 53,98 \\
\hline \multicolumn{3}{|c|}{ Características Hídricas } \\
\hline \multicolumn{3}{|l|}{ Umidade (\%) } \\
\hline Natural & 3,46 & 2,82 \\
\hline $10 \mathrm{kPa}$ & 29,11 & 26,37 \\
\hline $33 \mathrm{kPa}$ & 23,85 & 22,36 \\
\hline $100 \mathrm{kPa}$ & 18,44 & 16,18 \\
\hline $500 \mathrm{kPa}$ & 12,18 & 11,10 \\
\hline $1000 \mathrm{kPa}$ & 11,43 & 10,38 \\
\hline $1500 \mathrm{kPa}$ & 11,42 & 10,35 \\
\hline Água disponível (33-1500kPa) & 12,43 & 12,01 \\
\hline
\end{tabular}


Tabela 2. Características químicas do solo cultivado com goiabeira cultivar Paluma, com quatro anos de idade. Itaporanga, PB, 2005

\begin{tabular}{|c|c|c|}
\hline Características Químicas & $\begin{array}{c}\text { Profundidade } \\
(0-0,20 \mathrm{~m})\end{array}$ & $\begin{array}{l}\text { Profundidade } \\
(0,20-0,40 \mathrm{~m})\end{array}$ \\
\hline Cálcio $\left(\mathrm{cmol}_{\mathrm{c}} \mathrm{kg}^{-1}\right)$ & 6,80 & 7,20 \\
\hline Magnésio $\left(\mathrm{cmol}_{\mathrm{c}} \mathrm{kg}^{-1}\right)$ & 7,68 & 7,88 \\
\hline Sodio $\left(\mathrm{cmol}_{\mathrm{C}} \mathrm{kg}^{-1}\right)$ & 1,87 & 2,05 \\
\hline Potássio $\left(\mathrm{cmol}_{\mathrm{c}} \mathrm{kg}^{-1}\right)$ & 0,38 & 0,32 \\
\hline $\mathrm{S}\left(\mathrm{cmol}_{\mathrm{c}} \mathrm{kg}^{-1}\right)$ & 16,73 & 17,45 \\
\hline Hidrogênio $\left(\mathrm{cmol}_{\mathrm{c}} \mathrm{kg}^{-1}\right)$ & 0,00 & 0,00 \\
\hline Alumínio $\left(\mathrm{cmol}_{\mathrm{c}} \mathrm{kg}^{-1}\right)$ & 0,00 & 0,00 \\
\hline $\mathrm{T}\left(\mathrm{cmol}_{\mathrm{c}} \mathrm{kg}^{-1}\right)$ & 16,73 & 17,45 \\
\hline Carbonato de cálcio & Presença & Presença \\
\hline Carbono orgânico (g kg-1) & 0,60 & 0,30 \\
\hline Matéria orgânica $\left(\mathrm{g} \mathrm{kg}^{-1}\right)$ & 1,03 & 0,65 \\
\hline Nitrogênio $\left(\mathrm{g} \mathrm{kg}^{-1}\right)$ & 0,06 & 0,03 \\
\hline Fósforo (mg kg-1) & 14,55 & 11,37 \\
\hline $\mathrm{pH}$ em $\mathrm{H}_{2} \mathrm{O}(1: 2,5)$ & 8,07 & 7,40 \\
\hline Condutividade elétrica ( $\mathrm{dS} \mathrm{m}^{-1}$ ) & 0,30 & 0,33 \\
\hline
\end{tabular}

em quatro níveis: $\mathrm{L}_{1}=0,60 \mathrm{~L}_{\mathrm{b}}, \mathrm{L}_{2}=1,00 \mathrm{~L}_{\mathrm{b}}, \mathrm{L}_{3}=1,40 \mathrm{~L}_{\mathrm{b}}$ e $\mathrm{L}_{4}=1,80 \mathrm{~L}_{\mathrm{b}}$, em mm, e b) nitrogênio $(\mathrm{N})$, em quatro níveis: $\mathrm{N}_{1}=50, \mathrm{~N}_{2}=100, \mathrm{~N}_{3}=150$ e $\mathrm{N}_{4}=200 \mathrm{~kg} \mathrm{ha}^{-1}$, aplicados juntamente com a água de irrigação.

Os tratamentos lâmina total de água (L) e adubação nitrogenada (N) foram distribuídos em blocos casualizados, em esquema fatorial 4 x 4, com quatro repetições. Utilizou-se do sistema de irrigação por microaspersão, vazão nominal de $50 \mathrm{~L} \mathrm{~h}^{-1}$, pressão de serviço $250 \mathrm{kPa}$, raio molhado de $2,5 \mathrm{~m}$ e espaçamento $6 \times 5 \mathrm{~m}$.

A lâmina bruta de irrigação foi aplicada diariamente logo após poda de frutificação, com duração de 198 dias, calculada pela Equação 1.

$$
\mathrm{L}_{\mathrm{b}}=\frac{\text { Et. Kt. Kc. Ks }}{\mathrm{Ef}}-\mathrm{Pe}
$$

em que: $\mathrm{L}_{\mathrm{b}}$ - lâmina bruta de irrigação, em mm; Et - evaporação diária do tanque classe $\mathrm{A}$, em $\mathrm{mm}$; Kt - coeficiente de tanque $(0,80)$; Kc - coeficiente da cultura (Tabela 3); Ks - coeficiente de área molhada pelo emissor $(0,65)$; Pe - precipitação efetiva e Ef - eficiência de irrigação $(0,84)$.

Tabela 3. Fase fenológica da goiabeira cultivar Paluma e coeficiente de cultivo $(\mathrm{Kc})$

\begin{tabular}{clcc}
\hline Fases & Período de Desenvolvimento & $\begin{array}{c}\text { Número de dias } \\
\text { (acumulado) }\end{array}$ & Kc (médio) \\
\hline F1 & Brotação,crescimento Vegetativo & 63 & 0,68 \\
F2 & $\begin{array}{l}\text { Crescimento vegetativo, floração, } \\
\text { queda de fruto }\end{array}$ & 109 & 0,76 \\
F3 & Crescimento do fruto & 172 & 0,71 \\
F4 & Maturação e colheita & 200 & 0,62 \\
\hline
\end{tabular}

Fonte: Ferreira (2004)

Como fonte de nitrogênio utilizaram-se nitrato de potássio e uréia aplicados via água de irrigação, três vezes por semana durante o ciclo da cultura, no total de 80 aplicações.

O tempo de irrigação para se aplicar a lâmina bruta $\mathrm{L}_{\mathrm{b}}$ foi determinado pela Equação 2.

$$
\mathrm{T}_{\mathrm{i}}=\frac{\mathrm{L}_{\mathrm{b}} \cdot \mathrm{E}_{1} \cdot \mathrm{E}_{2}}{\mathrm{Q}}
$$

em que: $T_{i}$ - tempo de irrigação, em $h ; E_{1}$ - espaçamento entre emissores, $\mathrm{m}$; $\mathrm{E}_{2}$ - espaçamento entre plantas, $\mathrm{m}$ e $\mathrm{Q}$ - vazão do emissor, $\mathrm{L} \mathrm{h} \mathrm{h}^{-1}$

Para análise econômica utilizaram-se os seguintes valores: preço do produto (Py) de $\mathrm{R} \$ 0,50 \mathrm{~kg}^{-1}$ (produto vendido na propriedade); o custo da água $\left(\mathrm{C}_{\mathrm{L}}\right)$ foi calculado com base no preço de uma hora de funcionamento do sistema de irrigação, para aplicação da vazão necessária para um hectare, cujo valor foi de $\mathrm{R} \$ 0,65 \mathrm{~mm}^{-1} \mathrm{ha}^{-1}$; o custo do nitrogênio $\left(\mathrm{C}_{\mathrm{N}}\right)$ se compunha da aquisição do fertilizante no mercado local, o qual foi de $\mathrm{R} \$ 1,15 \mathrm{~kg}^{-1}$, enquanto o custo de produção para o quarto ano $\left(\mathrm{C}_{\mathrm{P}}\right)$ compreende todos os custos de produção, excetuando-se os diretamente relacionados com os fatores estudados (água e/ou adubação nitrogenada), o qual foi de R\$2.446,00 ha-1, Tabela 4.

Tabela 4. Custo de produção $\left(C_{p}\right)$ para 1,0 ha de goiaba, cultivar Paluma, com quatro anos de idade, irrigada por microaspersão. Itaporanga, PB, 2005

\begin{tabular}{lccrr}
\hline Discriminação & Unid. & $\begin{array}{c}\text { Valor unitário } \\
\text { (R\$) }\end{array}$ & Quant. & $\begin{array}{r}\text { Valor total } \\
\text { (R\$) }\end{array}$ \\
1. Tratos culturais & & & & $1.360,00$ \\
- Capinas & $H / D^{*}$ & 10,00 & 20 & 200,00 \\
- Roçagem & $H / M^{* *}$ & 35,00 & 08 & 280,00 \\
- Adubação (fósforo) & $H / D$ & 10,00 & 08 & 80,00 \\
- Aplicação defensivos & $H / D$ & 10,00 & 15 & 150,00 \\
- Manejo Irrigação Fertirrigação & $\mathrm{H} / \mathrm{D}$ & 10,00 & 40 & 400,00 \\
- Poda de Produção & $\mathrm{H} / \mathrm{D}$ & 10,00 & 25 & 250,00 \\
2. Colheita & $\mathrm{H} / \mathrm{D}$ & 10,00 & 25 & 250,00 \\
3. Insumos & & & & 786,00 \\
- Nitrato de Potássio & $\mathrm{Kg}$ & 0,60 & 200 & 120,00 \\
- Superfosfato simples & $\mathrm{Kg}$ & 0,40 & 200 & 80,00 \\
- Inseticidas/fungicidas & $\mathrm{Kg} \mathrm{L}-1$ & 55,40 & 10 & 554,00 \\
\hline - Espalhante adesivo & $\mathrm{L}$ & 8,00 & 04 & 32,00 \\
TOTAL (CP) & & & & $2.446,00$ \\
\hline * Homem dia; ** Hora máquina & & & &
\end{tabular}

A amortização anual do investimento foi estimada pela Equação 3, cujo valor foi de $\mathrm{R} \$ 885,62 \mathrm{ha}^{-1}$; o custo fixo $\left(\mathrm{C}_{\mathrm{F}}\right)$ corresponde ao somatório dos custos de produção e da amortização anual dos investimentos, cujo valor foi $\mathrm{R} \$ 3.331,62 \mathrm{ha}^{-1}$.

$$
\mathrm{A}=\mathrm{Io} . \mathrm{FRC}
$$

em que: A é a amortização anual dos investimentos no sistema de irrigação e na terra $\left(\mathrm{R} \$ \mathrm{ha}^{-1}\right)$, Io é o investimento no sistema de irrigação e na terra (R\$) e FRC é fator de recuperação do capital, calculado pela Eq. 4.

$$
F R C=\frac{i(1+i)^{n}}{(1+i)^{n}-1}
$$

em que: i é a taxa real anual de juros (decimal) e n é o 
número de anos para quitar o investimento ou vida útil dos equipamentos.

\section{RESULTADOS E DISCUSSÃO}

Conforme a análise de variância dos dados (Tabela 5) a produtividade da goiabeira foi influenciada, em nível de $1 \%$ de probabilidade, tanto pelo nível de nitrogênio como pelas lâminas totais de água aplicada. Verificaram-se, também, efeitos significativos para as interações entre os fatores estudados, indicando dependência entre eles.

Tabela 5. Resumo da ANAVA e médias de interação da produtividade da goiabeira em função dos níveis de nitrogênio Produto e das lâminas totais de água (irrigação + precipitação). Itaporanga, PB, 2005

\begin{tabular}{|c|c|c|c|c|}
\hline Fontes de variação & & G.L. & \multicolumn{2}{|c|}{ Quadrado Médio (QM) } \\
\hline Nitrogênio (N) & & 3 & \multicolumn{2}{|c|}{$1108,3278^{* *}$} \\
\hline Lâminas Totais (L) & & 3 & \multicolumn{2}{|c|}{$159,8006^{* *}$} \\
\hline$N \times L$ & & 9 & \multicolumn{2}{|c|}{$32,1235^{*}$} \\
\hline Blocos & & 3 & \multicolumn{2}{|c|}{$36,3772^{*}$} \\
\hline Resíduo & & 45 & \multicolumn{2}{|c|}{11,9062} \\
\hline CV(\%) & & 12,83 & & \\
\hline \multicolumn{5}{|c|}{ Médias de Interação } \\
\hline \multirow{2}{*}{$\begin{array}{l}\text { Lâminas totais } \\
(\mathrm{mm})\end{array}$} & \multicolumn{4}{|c|}{ Níveis de nitrogênio $\left(\mathrm{kg} \mathrm{ha}^{-1}\right)$} \\
\hline & $N_{1}(50)$ & $\mathrm{N}_{2}(\mathbf{1 0 0 )}$ & $\mathrm{N}_{3}(150)$ & $\mathrm{N}_{4}(\mathbf{2 0 0})$ \\
\hline $\mathrm{L}_{1}(1.144)$ & $14.286,9$ & $23.134,3$ & $23.914,3$ & $29.490,3$ \\
\hline $\mathrm{L}_{2}(1.465)$ & $14.625,8$ & $25.664,0$ & $27.666,0$ & $38.355,8$ \\
\hline $\mathrm{L}_{3}(1.785)$ & $17.903,0$ & $29.353,5$ & $30.076,0$ & 43583,5 \\
\hline$\underline{\mathrm{L}_{4}(2.106)}$ & $21.092,5$ & $22.558,5$ & $31.227,5$ & $37.086,5$ \\
\hline
\end{tabular}

* Significativo a $5 \%$ pelo teste $\mathrm{F}$; ${ }^{* *}$ Significativo a $1 \%$ pelo teste $\mathrm{F}$

Uma análise quantitativa da função de resposta da goiabeira à ação simultânea dos fatores lâminas de água e níveis de nitrogênio, foi mais significativamente representada pela regressão quadrática indicada na Eq. 5 e na Figura 1.

$$
\begin{aligned}
\mathrm{Y}(\mathrm{L}, \mathrm{N})= & -92361,763+119,29 \mathrm{~L}-0,0344 \mathrm{~L}^{2} \\
& +287,56 \mathrm{~N}-0,8676 \mathrm{~N}^{2}+0,0025 \mathrm{LN}
\end{aligned}
$$

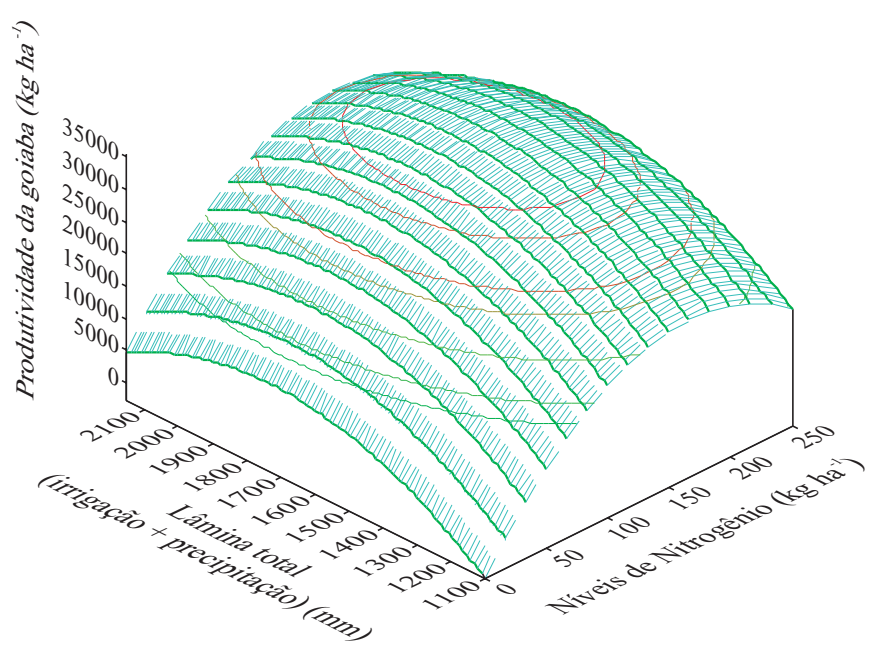

Figura 1. Superfície de resposta da goiabeira ás lâminas de água e níveis de nitrogênio. Itaporanga, PB. 2005
Observa-se na Figura 1 rendimento máximo da goiabeira de $35.607 \mathrm{~kg} \mathrm{ha}^{-1}$, obtido com a combinação da lâmina de água de 1.740 mm e do nível de nitrogênio de $68 \mathrm{~kg} \mathrm{ha}^{-1}$.

\section{Produtividade de máximo rendimento físico}

O rendimento físico foi obtido da a Eq. 5 em relação a cada fator obtendo-se as Equações 6 e 7 (Aguiar, 2005; Frizzone, 1993).

$$
\begin{aligned}
& \frac{\partial \mathrm{Y}}{\partial \mathrm{L}}=119,29-0,0688 \mathrm{~L}+0,0025 \mathrm{~N}=0 \\
& \frac{\partial \mathrm{Y}}{\partial \mathrm{N}}=287,56-1,7352 \mathrm{~N}+0,0025 \mathrm{~L}=0
\end{aligned}
$$

Apresentam-se, na Tabela 6, os pontos máximos de lâmina total de água $\left(\mathrm{L}_{\mathrm{m}}\right)$ e de níveis de nitrogênio $\left(\mathrm{N}_{\mathrm{m}}\right)$ encontrando-se, também, os dados de produtividade máxima da goiabeira ( $\mathrm{kg} \mathrm{ha}^{-1}$ ) estimados pela Equação 5 e a respectiva receita líquida obtida pela Eq. 8.

$$
\mathrm{RL}=\mathrm{P}_{\mathrm{y}} \mathrm{Y}-\mathrm{C}_{\mathrm{L}} \mathrm{L}-\mathrm{C}_{\mathrm{N}} \mathrm{N}-\mathrm{C}_{\mathrm{F}}
$$

\section{Produtividade que maximiza a receita líquida}

Os valores quantitativos referentes à água ( $\left.\mathrm{L}^{*}\right)$ e nitrogênio $\left(\mathrm{N}^{*}\right)$ através dos quais se detém a máxima receita líquida, e se considerando não haver restrição quanto aos recursos financeiros disponíveis para as suas aquisições, são obtidos derivando-se a Equação 5 em relação aos fatoes L e $\mathrm{N}$ e os igualando, à relação entre os preços unitários da água e da goiaba $\left(\mathrm{C}_{\mathrm{L}} / \mathrm{Py}=1,3\right)$ e a relação entre os preços unitários do nitrogênio e da goiaba $\left(\mathrm{C}_{\mathrm{N}} / \mathrm{Py}=2,3\right)$, respectivamente, obtendo-se as Equações 9 e 10 (Aguiar, 2005; Frizzone, 1993).

A solução de $\mathrm{L}^{*}$ e $\mathrm{N}^{*}$, a produtividade que maximiza a receita líquida $\mathrm{Y}\left(\mathrm{L}^{*}, \mathrm{~N}^{*}\right)$ calculada pela Eq. 5, e a respectiva receita líquida $\mathrm{R}_{\mathrm{L}}\left(\mathrm{L}^{*}, \mathrm{~N}^{*}\right)$ calculada pela Eq. 8, são apresentadas na Tabela 6 .

$$
\begin{aligned}
& \frac{\partial \mathrm{Z}}{\partial \mathrm{L}}=119,29-0,0688 \mathrm{~L}+0,0025 \mathrm{~N}=1,3 \\
& \frac{\partial \mathrm{Z}}{\partial \mathrm{L}}=119,29-0,0688 \mathrm{~L}+0,0025 \mathrm{~N}=1,3
\end{aligned}
$$

A Tabela 6 apresenta também uma análise de desempenho econômico para a combinação de lâminas de irrigação (L) e níveis de nitrogênio $(\mathrm{N})$ estudados neste trabalho. De acordo com a tabela, a produção de máximo rendimento físico $\left(\mathrm{Y}\left(\mathrm{L}_{\mathrm{m}}, \mathrm{N}_{\mathrm{m}}\right)\right)$, estimada em $35.607 \mathrm{~kg} \mathrm{ha}^{-1}$ de goiaba, ocorreu com a combinação $\mathrm{L}_{\mathrm{m}}=1.740 \mathrm{~mm}$ de água e $\mathrm{N}_{\mathrm{m}}=168 \mathrm{~kg} \mathrm{ha}^{-1}$ de $\mathrm{N}$; já a produção que maximiza a receita líquida (Y(L*, $\left.\mathrm{N}^{*}\right)$ ), estimada em $35.594 \mathrm{~kg} \mathrm{ha}^{-1}$, se deu com a combinação $\mathrm{L}^{*}=1.721 \mathrm{~mm}$ de água e $\mathrm{N}^{*}=167 \mathrm{~kg} \mathrm{ha}^{-1}$ de $\mathrm{N}$, as quais proporcionaram uma receita líquida de: $\mathrm{R}_{\mathrm{L}}\left(\mathrm{L}_{\mathrm{m}} ; \mathrm{N}_{\mathrm{m}}\right)=$ $\mathrm{R} \$ 13.147,68$ ha $^{-1}$ e $\mathrm{R}_{\mathrm{L}}\left(\mathrm{L}^{*} ; \mathrm{N}^{*}\right)=13.154,68 \mathrm{ha}^{-1}$.

A produção que maximiza a receita líquida é $0,036 \%$ menor que a produção de máximo rendimento físico; no entanto, esta proporcionou uma receita líquida 0,05\% superior àquela. 
Permanecendo constante a lâmina total de água e variando os níveis de nitrogênio observa-se, na Tabela 6, que a produtividade da goiabeira e respectiva receita líquida são crescentes de $\mathrm{N}_{1}$ até $\mathrm{N}_{3}$ e decrescentes de $\mathrm{N}_{3}$ a $\mathrm{N}_{4}$; com este mesmo raciocínio em relação aos níveis de nitrogênio como constantes e se variando as lâminas totais de água, nota-se que a produtividade e a receita líquida aumentam de $\mathrm{L}_{1}$ até $\mathrm{L}_{3}$ e diminuem de $\mathrm{L}_{3}$ a $\mathrm{L}_{4}$.

A produtividade obtida com a combinação $\mathrm{L}_{3} \mathrm{~N}_{3}$ (35.247 $\mathrm{kg} \mathrm{ha}^{-1}$ ) proporcionou uma receita líquida de $\mathrm{R} \$ 12.959,13 \mathrm{ha}^{-1}$, equivalente ao acréscimo de 81,3\% em relação à receita líquida de $\mathrm{R} \$ 7.149,13$ ha $^{-1}$ obtida com $\mathrm{L}_{3}$ $\mathrm{N}_{1}$ (23.970 kg ha), 16,3\% em relação à receita líquida de $\mathrm{R} \$ 11.138,63 \mathrm{ha}^{-1}$ correspondente a $\mathrm{L}_{3} \mathrm{~N}_{2}$ (31.491 $\mathrm{kg} \mathrm{ha}^{-1}$ ) e 2,8\% em relação à receita líquida de $\mathrm{R} \$ 12.610,63 \mathrm{ha}^{-1}$ para com $\mathrm{L}_{3} \mathrm{~N}_{4}$ (34.665 kg ha-1).

Tabela 6. Análise econômica para as lâminas totais de água que maximizam o rendimento físico $(\mathrm{Lm})$, a receita líquida $\left(\mathrm{L}^{*}\right)$ para a relação de custo $C_{1} / P y=1,3$. Itaporanga, $P B, 2005$

\begin{tabular}{|c|c|c|}
\hline $\begin{array}{l}\text { Parâmetros: } \\
\text { L(mm); N(kg ha-1) }\end{array}$ & $\begin{array}{l}\text { Produtividade (Y) } \\
\qquad\left(\mathrm{kg} \mathrm{ha}^{-1}\right)\end{array}$ & $\begin{array}{c}\text { Receita Líquida }\left(R_{L}\right) \\
\left(\mathbf{R} \$ \mathbf{h a}^{-1}\right)\end{array}$ \\
\hline $\mathrm{L}_{\mathrm{m}} ; \mathrm{N}_{\mathrm{m}}=1.740 ; 168$ & 35.607 & $13.147,68$ \\
\hline$L^{*} ; N^{*}=1.721 ; 167$ & 35.594 & $13.154,68$ \\
\hline $\mathrm{L}_{1} ; \mathrm{N}_{1}=1.144 ; 50$ & 11.437 & $1.585,78$ \\
\hline $\mathrm{L}_{1} ; \mathrm{N}_{2}=1.144 ; 100$ & 19.451 & $5.535,28$ \\
\hline $\mathrm{L}_{1} ; \mathrm{N}_{3}=1.144 ; 150$ & 23.127 & $7.315,78$ \\
\hline $\mathrm{L}_{1} ; \mathrm{N}_{4}=1.144 ; 200$ & 22.465 & $6.927,28$ \\
\hline $\mathrm{L}_{2} ; \mathrm{N}_{1}=1.465 ; 50$ & 20.960 & $6.138,63$ \\
\hline $\mathrm{L}_{2} ; \mathrm{N}_{2}=1.465 ; 100$ & 29.014 & $10.108,13$ \\
\hline $\mathrm{L}_{2} ; \mathrm{N}_{3}=1.465 ; 150$ & 32.730 & $11.908,63$ \\
\hline $\mathrm{L}_{2} ; \mathrm{N}_{4}=1.465 ; 200$ & 32.108 & $11.540,13$ \\
\hline $\mathrm{L}_{3} ; \mathrm{N}_{1}=1.785 ; 50$ & 23.397 & $7.149,13$ \\
\hline $\mathrm{L}_{3} ; \mathrm{N}_{2}=1.785 ; 100$ & 31.491 & $11.138,63$ \\
\hline $\mathrm{L}_{3} ; \mathrm{N}_{3}=1.785 ; 150$ & 35.247 & $12.959,13$ \\
\hline $\mathrm{L}_{3} ; \mathrm{N}_{4}=1.785 ; 200$ & 34.665 & $12.610,63$ \\
\hline $\mathrm{L}_{4} ; \mathrm{N}_{1}=2.106 ; 50$ & 18.763 & $4.623,48$ \\
\hline $\mathrm{L}_{4} ; \mathrm{N}_{2}=2.106 ; 100$ & 26.897 & $8.632,98$ \\
\hline $\mathrm{L}_{4} ; \mathrm{N}_{3}=2.106 ; 150$ & 30.694 & $10.473,98$ \\
\hline $\mathrm{L}_{4} ; \mathrm{N}_{4}=2.106 ; 200$ & 30.152 & $10.145,48$ \\
\hline
\end{tabular}

Também se observa, na Tabela 6, a tendência de acréscimo na receita líquida nas combinações $\mathrm{L}_{1} \mathrm{~N}_{3}, \mathrm{~L}_{2} \mathrm{~N}_{3}$ e $\mathrm{L}_{4} \mathrm{~N}_{3}$ em comparação com essas lâminas e os outros níveis de nitrogênio. Como se observa, a combinação de lâminas com o nível de nitrogênio $\mathrm{N}_{3}$ (150 $\mathrm{kg} \mathrm{ha}^{-1}$ ) proporcionou grande acréscimo na receita líquida em relação ao nível $\mathrm{N}_{1}\left(50 \mathrm{~kg} \mathrm{ha}^{-1}\right)$, evidenciando que o déficit de nitrogênio reduziu a produtividade da goiabeira; por outro lado, quanto a $\mathrm{N}_{4}\left(200 \mathrm{~kg} \mathrm{ha}^{-1}\right)$, embora o acréscimo na receita líquida tenha sido pequeno, evidencia-se que o excesso de nitrogênio também proporcionou redução na produtividade da goiabeira.

\section{Combinação dos fatores que resultam no custo mínimo}

Uma vez delimitada a região de produção racional, é oportuno saber quanto se deve gastar com os dois fatores de produção para se atingir determinado nível de produtividade ao menor custo possível. Conhecendo-se as diferentes combina- ções dos dois fatores substituíveis, que produzem a mesma quantidade do produto, pode-se determinar a combinação de custo mínimo, Equação 11 (Aguiar, 2005).

$$
\mathrm{L}=\frac{\mathrm{C}}{\mathrm{C}_{\mathrm{L}}}-\frac{\mathrm{C}_{\mathrm{N}}}{\mathrm{C}_{\mathrm{L}}} \mathrm{N}
$$

em que: $\mathrm{C}_{\mathrm{N}} / \mathrm{C}_{\mathrm{L}}$ é a declividade da reta; $\mathrm{C}$ - capital empregado, em $\mathrm{R} \$ \mathrm{C}_{\mathrm{L}}$ - custo da água, em $\mathrm{R} \$ \mathrm{C}_{\mathrm{N}}$ - custo do nitrogênio, em R\$.

Na Tabela 7 constam os custos e receitas para as combinações de água e nitrogênio que resultam em custo mínimo, ou máxima receita líquida, para as produtividades entre 14.000 a $34.000 \mathrm{~kg} \mathrm{ha}^{-1}$ de goiaba, para a relação de custo $\mathrm{C}_{\mathrm{N}} / \mathrm{C}_{\mathrm{L}}=1,769$.

Tabela 7. Combinações de lâminas de água (L) e doses de nitrogênio (N) que resultam em custo mínimo para produtividade de goiaba de 14.000 a $34.000 \mathrm{~kg} \mathrm{ha}^{-1}$ para a relação de custo $C_{N} / C_{L}=1,769$. Itaporanga, PB, 2005

\begin{tabular}{|c|c|c|c|c|c|}
\hline \multirow{2}{*}{$\begin{array}{l}\text { Produtividade } \\
\text { da goiabeira } \\
\left(\mathrm{kg} \mathrm{ha}^{-1}\right)\end{array}$} & \multicolumn{2}{|c|}{ Insumos } & $\begin{array}{l}\text { Custo dos } \\
\text { Insumos }\end{array}$ & Custo fixo & $\begin{array}{l}\text { Receita } \\
\text { Líquida }\end{array}$ \\
\hline & $\mathrm{L}(\mathrm{mm})$ & $N\left(k^{\prime} h^{-1}\right)$ & & (R\$ ha-1) & \\
\hline 14.000 & 1.169 & 58 & 826,55 & $3.331,62$ & $2.841,83$ \\
\hline 20.000 & 1.250 & 76 & 899,90 & $3.331,62$ & $5.768,48$ \\
\hline 26.000 & 1.358 & 95 & 991,95 & $3.331,62$ & $8.676,43$ \\
\hline 30.000 & 1.450 & 112 & $1.071,30$ & $3.331,62$ & 10.597 .08 \\
\hline 32.000 & 1.510 & 123 & $1.122,95$ & $3.331,62$ & $11.545,43$ \\
\hline 34.000 & 1.600 & 136 & $1.196,40$ & $3.331,62$ & $12.471,98$ \\
\hline
\end{tabular}

Observa-se, na Tabela 7, incremento de 5,5\% na receita líquida ( $\mathrm{R} \$ 12.471,68 \mathrm{ha}^{-1}$ ) proporcionado pela combinação $\mathrm{L}=1.600 \mathrm{~mm}$ e $\mathrm{N}=136 \mathrm{~kg} \mathrm{ha}^{-1}$, atingindo rendimento de $34.000 \mathrm{~kg} \mathrm{ha}^{-1}$ em relação à receita líquida (R\$13.154,68 $\mathrm{kg} \mathrm{ha}^{-1}$ ) proporcionada pela combinação $\mathrm{L}^{*}$ e $N^{*}$ (35.594 $\left.\mathrm{kg} \mathrm{ha}^{-1}\right)$.

\section{Produto físico marginal}

O produto físico marginal, ou produtividade marginal pode ser expresso pela quantidade adicionada ao produto total, quando uma unidade do fator variável é utilizada (Aguiar, 2005).

$\mathrm{O}$ produto físico marginal da água $\left(\mathrm{PFM}_{\mathrm{L}}\right)$, medido em quilograma de fruto de goiaba por milímetro de água por hectare, é calculado derivando-se a Equação 5, em função da lâmina de água, cujo valor está apresentado na Tabela 8. O produto físico marginal mostra o incremento no rendimento ao se usar uma unidade a mais do fator considerado; por exemplo, empregando- se $150 \mathrm{~kg} \mathrm{ha}^{-1}$ de nitrogênio e $1.465 \mathrm{~mm}$ de água, o rendimento aumenta $18,87 \mathrm{~kg} \mathrm{ha}^{-1}$ para cada milímetro de água aplicado, conforme a mesma tabela.

Observa-se, na Tabela 8, quando se aumenta a lâmina total de água de $L_{1}$ para $L_{2}$, em todos os níveis de nitrogênio, que o produto marginal diminui; já na aplicação das lâminas $\mathrm{L}_{3}$ e $\mathrm{L}_{4}$, a produtividade marginal passa a ser negativa, ou seja, o rendimento inicia uma fase de retorno decrescente.

Verifica-se, ainda, para todos os níveis de nitrogênio, que 
Tabela 8. Produto físico marginal da água em cada nível de nitrogênio, para as lâminas totais de água (irrigação + precipitação). Itaporanga, PB, 2005

\begin{tabular}{ccccc}
\hline Lâminas totais de água & \multicolumn{4}{c}{ Níveis de nitrogênio (kg ha-1) } \\
\cline { 2 - 5 }$(\mathbf{m m})$ & $\mathbf{N}_{\mathbf{1}} \mathbf{( 5 0 )}$ & $\mathbf{N}_{\mathbf{2}} \mathbf{( 1 0 0 )}$ & $\mathbf{N}_{\mathbf{3}}(\mathbf{1 5 0})$ & $\mathbf{N}_{\mathbf{4}} \mathbf{( 2 0 0 )}$ \\
$\mathrm{L}_{1}(1.144)$ & 40,71 & 40,83 & 40,96 & 41,08 \\
$\mathrm{~L}_{2}(1.465)$ & 18,62 & 18,75 & 18,87 & 19,00 \\
$\mathrm{~L}_{3}(1.785)$ & $-3,39$ & $-3,27$ & $-3,14$ & $-3,02$ \\
$\mathrm{~L}_{4}(2.106)$ & $-25,48$ & $-25,35$ & $-25,23$ & $-25,10$ \\
\hline
\end{tabular}

a máxima produtividade de goiaba é alcançada quando a lâmina total aplicada se situa entre $L_{2}$ e $L_{3}$, uma vez que o produto físico marginal passa de positivo a negativo. A partir do momento em que o produto marginal da água se torna negativo, evidencia-se queda no rendimento com a respectiva aplicação de maiores lâminas de água, tornando-se antieconômicas as quantidades aplicadas desse fator.

\section{Taxa marginal de substituição}

A taxa marginal de substituição $\left(\mathrm{TMS}_{\mathrm{L} / \mathrm{N}}\right)$ de água por nitrogênio é a quantidade de água que deve substituir uma unidade do fator nitrogênio, de modo a manter o mesmo nível de rendimento (Aguiar, 2005; Frizzone, 1993).

Inicialmente, a TMS é negativa, indicando que a água está sendo substituída pelo nitrogênio em proporções decrescentes; a partir do momento em que ela se torna positiva, torna-se antieconômica a substituição da água por nitrogênio, ou seja, passaria a gastar mais no fator água a cada unidade acrescida do fator nitrogênio.

Observam-se, na Tabela 9, as taxas marginais de substituição $\left(\mathrm{TMS}_{\mathrm{L} / \mathrm{N}}\right)$ de lâminas de água por níveis de nitrogênio para níveis prefixados de produtividade de goiaba calculados pela Eq. 12 .

$$
\mathrm{TMS}_{\mathrm{L} / \mathrm{N}}=-\frac{\mathrm{PFM}_{\mathrm{N}}}{\mathrm{PFM}_{\mathrm{L}}}=-\frac{287,56-1,7352 \mathrm{PN}+0,0025 \mathrm{~L}}{119,29-0,0688 \mathrm{~L}+0,0025 \mathrm{~N}}
$$

Tabela 9. Taxas marginais de substituição $\left(\mathrm{TMS}_{\mathrm{L} / \mathrm{N}}\right)$ de lâmina de água por níveis de nitrogênio, para níveis prefixados de rendimento de goiaba. Itaporanga, PB, 2005

\begin{tabular}{|c|c|c|c|c|c|c|c|c|}
\hline \multirow{3}{*}{$\begin{array}{l}\text { Niveis } \\
\text { de N } \\
\text { kg ha-1 }^{-1}\end{array}$} & \multicolumn{2}{|c|}{$Y=20.000$} & \multicolumn{2}{|c|}{$Y=26.000$} & \multicolumn{2}{|c|}{$Y=30.000$} & \multicolumn{2}{|c|}{$Y=34.000$} \\
\hline & \multicolumn{8}{|c|}{$\left(\mathrm{kg} \mathrm{ha}^{-1}\right)$} \\
\hline & $\mathrm{L}(\mathrm{mm})$ & TMS & $\mathrm{L}(\mathrm{mm})$ & TMS & $\mathrm{L}(\mathrm{mm})$ & TMS & $\mathrm{L}(\mathrm{mm})$ & TMS \\
\hline 40 & 1538 & $-16,35$ & - & - & - & - & - & - \\
\hline 50 & 1418 & $-9,32$ & - & - & - & - & - & - \\
\hline 60 & 1338 & $-6,82$ & - & - & - & - & - & - \\
\hline 70 & 1278 & $-5,37$ & 1547 & $-13,03$ & - & - & - & - \\
\hline 80 & 1230 & $-4,35$ & 1449 & $-7,69$ & - & & - & - \\
\hline 100 & 1158 & $-2,93$ & 1335 & $-4,24$ & 1524 & $-8,02$ & - & - \\
\hline 120 & 1110 & $-1,90$ & 1269 & $-2,65$ & 1415 & $-3,73$ & - & - \\
\hline 140 & 1081 & $-1,05$ & 1230 & $-1,36$ & 1361 & $-1,85$ & 1576 & $-4,33$ \\
\hline 160 & 1067 & $-0,27$ & 1213 & $-0,36$ & 1338 & $-0,48$ & 1524 & $-0,93$ \\
\hline
\end{tabular}

De acordo com a Tabela 9, a taxa marginal de substituição de água por nitrogênio decresce em valor absoluto ao aumentar o nível de nitrogênio e diminui a lâmina total de água; nota-se também que, à medida que a produtividade de goiaba cresce, reduz-se o número de combinações possíveis para obtenção de um mesmo nível de rendimento; além disso, para todos os níveis de produtividade da goiabeira a taxa marginal de substituição se aproxima de zero, no nível de nitrogênio igual a $160 \mathrm{~kg} \mathrm{ha}^{-1}$ sendo, portanto, este nível, o limite de substituição de nitrogênio por lâmina de água e sempre que se utilizem maiores quantidades de nitrogênio são necessárias maiores quantidades de lâmina de água para se obter a mesma produtividade.

\section{Eficiência de uso da água}

A eficiência de uso da água (EUA) foi obtida pelo quociente entre a produtividade da cultura $\left(\mathrm{kg} \mathrm{ha}^{-1}\right)$ e o volume total de água aplicada durante o ciclo da cultura $\left(\mathrm{m}^{3} \mathrm{ha}^{-1}\right)$ e os valores são apresentados na Tabela 10.

Tabela 10. Eficiência de uso da água $\left(\mathrm{kg} \mathrm{m}^{-3}\right)$ em função de lâminas de água e níveis de nitrogênio aplicados. Itaporanga, PB, 2005

\begin{tabular}{cccccc}
\hline Lâminas totais & \multicolumn{5}{c}{ Níveis de nitrogênio $\left(\mathbf{k g ~ h a} \mathbf{~}^{\mathbf{1}}\right)$} \\
\cline { 2 - 6 } de água $\left(\mathbf{m}^{\mathbf{3}} \mathbf{h a}^{-1}\right)$ & $\mathbf{5 0}$ & $\mathbf{1 0 0}$ & $\mathbf{1 5 0}$ & $\mathbf{2 0 0}$ & Médias \\
11.440 & 1,25 & 2,02 & 2,09 & 2,58 & 1,98 \\
14.650 & 1,00 & 1,75 & 1,89 & 2,62 & 1,81 \\
17.850 & 1,00 & 1,64 & 1,68 & 2,44 & 1,69 \\
21.060 & 1,00 & 1,07 & 1,48 & 1,76 & 1,33 \\
\hline Médias & 1,06 & 1,62 & 1,79 & 2,35 & \\
\hline
\end{tabular}

Observa-se, na Tabela 10, que em média a eficiência do uso da água diminuiu com o aumento do volume de água aplicado e cresceu com o aumento dos níveis de nitrogênio. Dentro de cada nível de nitrogênio os valores médios da eficiência de uso da água aumentaram com o incremento dos níveis de nitrogênio até $\mathrm{N}_{3}\left(150 \mathrm{~kg} \mathrm{ha}^{-1}\right)$ decrescendo, em seguida, com $\mathrm{N}_{4}$; o maior valor de eficiência de uso da água pela cultura, $2,62 \mathrm{~kg} \mathrm{~m}^{-3}$, foi encontrado com a combinação $14.650 \mathrm{~m}^{3} \mathrm{ha}^{-1}$ e $200 \mathrm{~kg} \mathrm{ha}^{-1}\left(\mathrm{~L}_{3}\right.$ e $\mathrm{N}_{3}$ ) este valor, porem, é inferior ao obtido por Teixeira et al. (2003) e Moura (2005), que acusaram eficiência de 2,66 e 2,91 $\mathrm{kg} \mathrm{m}^{-3}$, em goiabeira cultivar Paluma, com dois anos e três meses e com quatro anos de idade, respectivamente.

\section{CONCLUSÕES}

1. O déficit e o excesso de água e nitrogênio provocaram redução na produtividade da goiabeira.

2. A resposta da goiabeira aos fatores lâmina de água (L) e adubação nitrogenada $(\mathrm{N})$, foi significativa, atingindo uma produtividade máxima de 43.583,5 $\mathrm{kg} \mathrm{ha}^{-1}$ com a combinação $\mathrm{L}=1.785 \mathrm{~mm}$ e $\mathrm{N}=200 \mathrm{~kg} \mathrm{ha}^{-1}$.

3. A produtividade máxima econômica foi estimada em $35.594 \mathrm{~kg} \mathrm{ha}^{-1}$ com a combinação L $=1.721$ e $\mathrm{N}=167 \mathrm{~kg} \mathrm{ha}^{-1}$ e receita líquida de $\mathrm{R} \$ 13.154,68 \mathrm{ha}^{-1}$.

4. A combinação dos fatores $1465 \mathrm{~mm}$ de água com $200 \mathrm{~kg} \mathrm{ha}^{-1}$ de nitrogênio resultou em uma eficiência de uso de água $2,62 \mathrm{~kg} \mathrm{~m}^{-3}$. 


\section{AGRADECIMENTOS}

Os autores agradecem ao Conselho Nacional de Desenvolvimento Científico e Tecnológico pelo apoio financeiro, do projeto: Manejo da fertirrigação em fruteiras tropicais através da irrigação localizada, proc. 475945/03-4, e à Empresa Paraibana de Pesquisa Agropecuária (EMEPA) pela área experimental e apoio nos trabalhos de campo.

\section{LITERATURA CITADA}

Aguiar, J. V. de. A função de produção na agricultura irrigada. Fortaleza: Imprensa Universitária, 2005. 196p.

Bassoi, L. H.; Teixeira, A. H. C.; Silva, J. A. M.; Silva, E. E. G. da; Targino, E. de L.; Maia, J. L. T; Ferreira, M. de N. L. Parâmetros para o manejo de irrigação da goiabeira no Vale de São Francisco. In: Congresso Brasileiro de Engenharia Agrícola, 31, 2002. Salvador. Anais... Salvador: SBEA/UFBA. 2002. CD-Rom.

CODEVASF - Companhia de Desenvolvimento do Vale São Francisco e Parnaiba. Cadastro frutícula do Vale do São Francisco. http//www.codevasf.gov.br. 15 Fev. 2005.

Ferreira, M. de N. L. Distribuição radicular e consumo de água de goiabeira (Psidium guajava L.) irrigada por microaspersão em Petrolina. Piracicaba: ESALQ/USP, 2004. 106p. Tese Doutorado

Frizzone, J. A. Funções de resposta das culturas à irrigação. Piracicaba: ESALQ/USP, 1993. 42p. Série Didática, 6

Gonzaga Neto, L. Goiaba: produção - aspectos técnicos. Embrapa Semi-Árido, Brasília: Embrapa Informações Tecnologias, 2001. 79p. Frutas do Brasil, 17
Lima, M. A. C. de; Assis, J. S. de; Gonzaga Neto, L. Caracterização dos frutos de goiabeira e seleção de cultivares na região do Submédio São Francisco. Revista Brasileira de Fruticultura, Jaboticabal, v.24, n.1, p.273-276, 2002.

Lopez, C. C. Fertirigação: Aplicação na horticultura. In: Folegatti, M. V.; Casarine, E.; Blanco, F. F.; Camponez do Brasil, R. P.; Resende, R. S. (coords). Fertirrigação: flores, frutas e hortaliças. Guaíba: Agropecuária, cap.8, p.269-288, 2001.

Martinez Júnior, M.; Pereira, F. M. Resposta da goiabeira a diferentes quantidades de NPK. In: Congresso Brasileiro de Fruticultura, 8, 1986. Brasília. Anais... Brasília: Embrapa/DDT, 1986. p.293-296.

Moura, M. S. B, de. Consumo hídrico, produtividade e qualidade do fruto da goiabeira irrigada no Submédio São Francisco. Campina Grande: UFCG 2005. 122p. Tese Doutorado

Papadopoulos, I. Tendências da fertirrigação: processos de transição na fertilização convencional para a fertirrigação. In: Folegatti, M. V.; Casarine, E.; Blanco, F. F.; Camponez do Brasil, R. P.; Resende, R. S. (coords). Fertirrigação: Flores, frutas e hortaliças. Guaíba: Agropecuária, cap.1, p.9-59, 2001.

Sousa, E. F, de. Funções de produção da cana-de-açúcar e da goiabeira em relação à irrigação. Campos dos Goytacazes: UENF, 1997. 119p. Tese Doutorado

Teixeira, A. H. de C.; Bassoi, L. H.; Reis, V. C. da S.; Silva, T. G. F. da; Ferreira, M. de N. L.; Maia, J. L. T. Estimativa do consumo hídrico da goiabeira, utilizando estações agrometeorológicas automática e convencional. Revista Brasileira de Fruticultura, Jaboticabal, v.25, n.3, p.457-460, 2003. 